\title{
Clinical characteristics of brain tumor- related epilepsy and factors influencing the identification of epilepsy-associated tumors
}

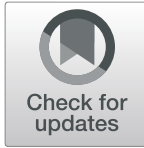

Xianwen Zhang ${ }^{1 \dagger}$, Liaoyuan Zheng ${ }^{2 \dagger}$, Jingfeng Duan ${ }^{1}$, Zongping Li $^{2}$ and Yufeng Tang ${ }^{{ }^{*}}$

\begin{abstract}
Background: To analyze the clinical features of brain tumor-related epilepsy (BTRE) and explore the factors influencing the identification of epilepsy-associated tumor (EAT), in order to advance the clinical understanding of BTRE and EAT.

Methods: Intracranial tumor origin and location as well as the type of epilepsy were retrospectively reviewed in 153 BTRE patients. The patients were further divided into the EAT and non-EAT groups, and comparisons were made for age, sex, tumor origin and location, and epilepsy type between the two groups.

Results: The 153 BTRE patients were divided into 78 cases with primary intracranial tumor and 75 cases with tumor originating from extracranial metastasis, according to the origin of tumor. According to the location of tumor, 116 cases had tumor lesions located in the brain parenchyma, and 37 cases had tumor lesions located in the meninges. Further, in the group with a brain parenchyma location, 77 cases had single lobular involvement, and 39 cases had multiple-lobular involvement; 84 cases had tumor lesions located in one hemisphere and 32 cases in both hemispheres. According to the type of epilepsy, 92 cases had generalized seizures, and 61 cases had focal seizures. The type of epilepsy did not significantly correlate with the origin of intracranial tumor, the location of tumor lesions (in brain parenchyma or meninges) $(P>0.05)$, or the hemispherical location (in one or two hemispheres) of lesions ( $P>0.05)$, but was significantly related with the lobular localization of lesions $(P<0.05)$. The 153 cases of BTRE consisted of 87 EAT and 66 non-EAT, with significant differences in the origin, location and type (being glioma/non-glioma) of tumor. Logistic regression analysis showed that the type of tumor (i.e. whether being glioma) served as an independent factor for EAT identification; the lower the World Health Organization grade of glioma, the more likely the EAT is to be diagnosed $(P<0.05)$.
\end{abstract}

Conclusion: The majority of BTRE patients in this study had tumors located in the brain parenchyma. In addition, the patients with generalized seizures outnumbered those with focal seizures, and the type of epilepsy was correlated with the lobular location of tumor lesions. The EATs are mostly low-grade gliomas.

Keywords: Brain tumor-related epilepsy, Epilepsy-associated tumor, Glioma, Epilepsy

\footnotetext{
* Correspondence: dryufeng@126.com

${ }^{+}$Xian-Wen Zhang and Liao-Yuan Zheng contributed equally to this work.

'Department of Neurology, Mianyang Central Hospital, Mianyang 621000,

China

Full list of author information is available at the end of the article
}

(c) The Author(s). 2020 Open Access This article is licensed under a Creative Commons Attribution 4.0 International License, which permits use, sharing, adaptation, distribution and reproduction in any medium or format, as long as you give appropriate credit to the original author(s) and the source, provide a link to the Creative Commons licence, and indicate if changes were made. The images or other third party material in this article are included in the article's Creative Commons licence, unless indicated otherwise in a credit line to the material. If material is not included in the article's Creative Commons licence and your intended use is not permitted by statutory regulation or exceeds the permitted use, you will need to obtain permission directly from the copyright holder. To view a copy of this licence, visit http://creativecommons.org/licenses/by/4.0/. 


\section{Background}

Epilepsy is a common clinical manifestation of intracranial tumor. About $25-60 \%$ of patients with intracranial tumor may develop symptoms of epilepsy. Brain tumor-related epilepsy (BTRE), as the term implies, represents the relations of two significant neurological subspecialties (neurooncology and epilepsy) and conditions (brain tumors and seizures) [1]. In BTRE, seizures may occur either as an initial symptom that leads to the diagnosis of tumor (seen in about $30-50 \%$ of patients) or during the course of the disease (seen in 10-30\% of patients) [2]. Some brain tumors grow relatively slowly and are not easy to found, with epilepsy as the primary and almost dominant symptom, thus they are called epilepsy-associated brain tumor or epilepsy-associated tumor (EAT). EAT is one type of BTRE, and is currently a common cause of focal epilepsy especially in children and young adults. EAT is clinically characterized by drug-resistant epilepsy and usually has a better prognosis after complete resection [3]. EAT with a history of drug-resistant seizures for over 2 years is called long-term epilepsy-associated tumor (LEAT)[4]. Currently, the risk factors, pathogenesis, types of seizure, diagnosis, and treatment of BTRE, as well as the influencing factors of EAT have not been studied extensively, and controversy remains among studies. In this study, we set out to retrospectively review the origin and location of intracranial tumor, the type of seizure, and the classification of tumor-related epilepsy in 153 patients with BTRE, analyze the clinical characteristics of BTRE, and determine the independent factors that influence EAT identification, in order to provide theoretical guidance for clinical management of BTRE and facilitate early detection and treatment of EAT.

\section{Participants and methods Participants}

One hundred and fifty-three BTRE patients (85 males and 68 females, age range $3-88$ years, average $48.67 \pm$ 16.58 years) who were diagnosed and treated in Mianyang Central Hospital of Sichuan Province from January 2010 to December 2017 were employed in this study, based on the following inclusion criteria: (1) diagnosed with epilepsy according to the definitions proposed by the International League Against Epilepsy in 2005[5]; (2) brain imaging (MRI or CT) suggesting intracranial tumor (including primary and secondary); and (3) histopathological evaluation of biopsy confirming the diagnosis of tumor. Patients with epilepsy caused by paraneoplastic syndrome, autoimmune encephalitis and radiation encephalopathy were excluded from the study.

\section{Methods}

Information on the gender, age, clinical symptoms, tumor origin, brain imaging and EEG of the patients was obtained by reviewing medical records or from patients and/or their family members at return visits. According to the preoperative brain imaging results, tumor boundaries were delineated and lesion locations were determined. The correlations of seizure type with the origin or location of intracranial tumor were analyzed. The epilepsy was classified as generalized and focal epilepsy according to The International League Against Epilepsy Classification of the Epilepsies in 2017 [6]. The 153 participants were divided into EAT and non-EAT groups according to whether epilepsy is the primary and main symptom, and the two groups were compared for seizure type, tumor origin, and tumor location to determine the independent factors for EAT identification.

\section{Statistical analysis}

Categorical variables were analyzed with the chi-square test. Comparisons between the EAT and non-EAT groups were performed with the independent sample $t$ test and chi-square test. The risk factors, confounders, and their interactions were analyzed with the logistic regression model, and odds ratios with $95 \%$ confidence intervals were calculated. All analyses were performed using the SPSS 21.0 software. A two-sided $P<0.05$ was considered as statistically significant.

\section{Results \\ Origin of intracranial tumor}

Of the 153 BTRE patients, 78 (51\%) patients had tumors originating from the primary intracranial tumor, including 57 cases of glioma, 16 cases of meningioma, and 1 case each for capillary hemangioblastoma, germ cell tumor, central nervous system malignant melanoma, primary central nervous system lymphoma, and dysembryoplastic neuroepithelial tumor. The other 75 cases (49\%) had tumors originating from extracranial tumor metastasis. The gliomas had the highest percent (37.3\%) among all types of origin (Table 1).

\section{Distribution of tumor lesions}

Of the 153 BTRE patients, 116 (75.8\%) had tumor lesions located in the brain parenchyma and 37 (24.2\%) had tumor lesions located in the meninges. Furthermore, of the patients with tumor lesion in the brain parenchyma, 77 (50.3\%) of them had single lobular location of tumor lesion, including 48 patients having tumor lesion in the frontal lobe, 16 in the temporal lobe, 11 in the parietal lobe, one in the cerebellum and one in the basal ganglia, while 39 (25.5\%) patients had multi-lobular location of tumor lesion. In addition, 84 (54.9\%) of the 116 patients had involvement of one hemisphere while 32 (20.9\%) of them had involvement of both hemispheres (Table 2). 
Table 1 Types of tumor origin in 153 cases of brain tumorrelated epilepsy

\begin{tabular}{lll}
\hline Origin & $\boldsymbol{n}$ & Percentage (\%) \\
\hline Primary intracranial tumor & & \\
Glioma & 57 & 37.3 \\
Meningioma & 16 & 10.5 \\
Capillary hemangioblastoma & 1 & 0.7 \\
Germ cell tumor & 1 & 0.7 \\
Central nervous system malignant melanoma & 1 & 0.7 \\
Primary central nervous system lymphoma & 1 & 0.7 \\
Dysembryoplastic neuroepithelial tumor & 1 & 0.7 \\
Metastasis & & \\
Lung cancer & 43 & 28.1 \\
Gastrointestinal cancer & 12 & 7.8 \\
Breast cancer & 5 & 3.3 \\
Hematologic malignancy & 4 & 2.6 \\
Genitourinary cancer & 3 & 2.0 \\
Tumor of female reproductive system & 2 & 1.3 \\
Malignant melanoma & 2 & 1.3 \\
Nasopharyngeal carcinoma & 1 & 0.7 \\
Unknown Origin & 3 & 2.0 \\
\hline & &
\end{tabular}

\section{Types of epilepsy}

Of the 153 BTRE patients, 92 (60.1\%) had generalized seizures, while 61 (39.9\%) had focal seizures. In particular, 23 cases had symptoms of automatisms, and their lesions were all located in the supratentorial lobe. The type of epilepsy did not significantly correlate with the origin of intracranial tumor, the location of tumor lesions (in brain parenchyma or meninges) $(P>0.05)$, or the hemispherical (one or two hemispheres) location of

Table 2 Tumor lesions locations in 153 cases of brain tumorrelated epilepsy

\begin{tabular}{llll}
\hline Location & & $\begin{array}{l}\text { Number of } \\
\text { patients }\end{array}$ & Percentage \\
\hline $\begin{array}{llll}\text { Brain parenchyma } \\
\text { Lobuar involvement }\end{array}$ & $\begin{array}{l}\text { Frontal lobe } \\
\text { Temporal } \\
\text { lobe }\end{array}$ & 48 & $31.4 \%$ \\
& $\begin{array}{l}\text { Parietal lobe } \\
\text { Cerebellum }\end{array}$ & 11 & $10.5 \%$ \\
& Basal ganglia & 1 & $7.2 \%$ \\
& Multiple lobes & 39 & $0.7 \%$ \\
Hemispherical & One & 84 & $0.7 \%$ \\
involvement & hemisphere & $25.5 \%$ \\
& $\begin{array}{l}\text { Both } \\
\text { hemispheres }\end{array}$ & 32 & $54.9 \%$ \\
Meninges & & 37 & $20.9 \%$ \\
\hline
\end{tabular}

lesions in brain parenchyma $(P>0.05)$, but was significantly related with the lobular localization of lesions $(P<0.05)$ (Tables 3 and 4$)$.

\section{Classification of EAT and non-EAT}

Of the 153 patients with BTRE, 87 had epilepsy as the primary and main symptom, and were assigned to the EAT group, while the remaining 66 patients were assigned to the non-EAT group. Among the 87 cases of EAT, there were two cases of LEAT, one case of dysembryoblastic neuroepithelial tumor found after a 17-year history of epilepsy, and one case of gemistocytic astrocytoma after a 4-year history of epilepsy. The EAT and non-EAT groups did not differ significantly in age $(t$ test), sex, type of seizure or the location of tumor lesions (in brain parenchyma or meninges) (chi-square test, $P>$ $0.05)$, but they were significantly different in the origin of tumor, the lobular and hemispheric locations of tumor, and the type of tumor (glioma/non-glioma) (chisquare test, $P<0.05$, Table 5 ).

Next, we established a logistic regression model using tumor origin, involvement of single or multiple lobes/ meninges, involvement of unilateral or bilateral hemispheres/meninges, and the type of tumor (whether being glioma, which can be further divided into World Health Organization [WHO] grades I-IV [7]) as independent variables, and whether being EAT as a dependent variable. The type of tumor (whether being glioma) was selected into the regression equation at $\alpha=0.05$. After correction of confounding factors, whether being glioma was determined to be the influencing factor for EAT $(P<0.05)$, and the lower the WHO grade of glioma, the more likely the EAT is to occur (Table 6).

\section{Discussion}

Brain tumor is a common cause of epilepsy. About 5\% of new epilepsy patients have brain tumor, and about $10 \%$ of epilepsy with focal seizures are caused by brain tumor [8]. BTRE is a multi-factorial condition with unclear physiological mechanisms. Current studies have indicated that epilepsy is associated with many factors, such as the histological type of intracranial tumor, the severity and location of tumor, genetic factors, the integrity of the blood-brain barrier, peritumoral morphological changes, changes in neurotransmitters, abnormal ion concentrations, and tissue hypoxia [9].

Although seizures may develop from almost all types of intracranial tumors (including primary and secondary brain tumors), the incidence of epilepsy varies among different histological types. For example, the incidence of epilepsy is $100 \%$ in dysembryoblastic neuroepithelial tumors, $25 \%$ in meningiomas, $10 \%$ in primary central nervous system lymphoma, and $20-35 \%$ in intracranial metastases [2]. About $20-45 \%$ of patients with glioma 
Table 3 Correlations of seizure type with tumor type

\begin{tabular}{lll}
\hline Seizure type & Primary & Metastasis \\
\hline Generalized $(n)$ & 51 & 41 \\
Focal $(n)$ & 27 & 34 \\
$P$ & 0.175 & \\
\hline
\end{tabular}

present with epilepsy as the first symptom at disease onset, and the other $15-30 \%$ of glioma patients develop epilepsy during the course of disease progression [10]. The incidence of epilepsy in glioma varies according to the grade of the tumor, with the WHO grade I-II gliomas more prone to epilepsy than WHO grade III-IV gliomas. The incidence of epilepsy is $70-80 \%$ in glioneuronal tumor, $60-75 \%$ in low-grade glioma (WHO grade I-II), and $25-60 \%$ in high-grade glioma (WHO grade III-IV) [11]. In general, the slowly-growing lowgrade tumors and intracranial multi-focal tumors have a higher propensity to develop epilepsy than the highergrade tumors and focal tumors, respectively [12]. Regardless of the type of intracranial tumor, patients with epilepsy as the first symptom at disease onset have a significantly increased risk of drug-refractory epilepsy [13]. In this study, 78 of the 153 BTRE patients were patients with primary tumor, and the other 75 patients had tumor from extracranial tumor metastases. A majority (57 cases, $73.1 \%$ ) of the primary tumors were gliomas, suggesting that patients with glioma are more likely to develop tumor-related epilepsy, which is consistent with previous reports. There is also a clear relationship between the location of the tumor and the occurrence of epilepsy. Studies have shown that the frontal lobe, temporal lobe, and parietal lobe are closely associated with language, cognition, and motor function $[14,15]$. Lesions in these areas can cause abnormal firing in these regions and thus seizures, and the occurrence of seizures may be further increased by glioma invasion into the cortex. Therefore, the incidence of epilepsy would be extremely high with a tumor location in the cortex, and the rate of seizures may be higher with locations of brain tumor in the frontal lobe, temporal lobe, and parietal lobe than in the occipital lobe. In contrast, patients with tumor lesions in the infratentorial or sellar region are less likely to develop epilepsy, except in the condition of

Table 4 Correlations of seizure type with tumor location

\begin{tabular}{llll}
\hline $\begin{array}{l}\text { Seizure } \\
\text { type }\end{array}$ & $\begin{array}{l}\text { Brain } \\
\text { parenchyma } \\
\text { / meninges }\end{array}$ & $\begin{array}{l}\text { Involvement } \\
\text { of one/both } \\
\text { hemispheres }\end{array}$ & $\begin{array}{l}\text { Frontal lobe/temporal } \\
\text { lobe/parietal lobe/ } \\
\text { cerebellum/ basal } \\
\text { ganglia/multiple lobes }\end{array}$ \\
\hline $\begin{array}{l}\text { Generalized } \\
(n)\end{array}$ & $68 / 24$ & $50 / 18$ & $28 / 7 / 4 / 1 / 1 / 31$ \\
Focal $(n)$ & $48 / 13$ & $34 / 14$ & $20 / 9 / 7 / 0 / 0 / 8$ \\
$P$ & 0.50 & 0.75 & 0.04 \\
\hline
\end{tabular}

hemispherical involvement [16]. Currently, few studies have focused on BTRE epilepsy. In this study, $60.1 \%$ of the patients had generalized seizures, while only $39.9 \%$ had focal seizures, including 23 patients having symptoms of automatisms with lesions all located in the supratentorial lobe. This suggests that a major proportion of BTRE may have a generalized origin. In addition, we found that the type of epilepsy did not have histological correlation, nor was it correlated with the location of the lesion (in the brain parenchyma or meninges), but it was related to the lobular localization of lesions. The lesions can be located in different lobes of the brain, accompanied by different types of epilepsy. Lesions in the frontal lobe tend to cause generalized seizure, while lesions in the temporal and parietal lobe are more likely to cause focal seizure.

Most cases of EAT have benign tumors that grow slowly with a long clinical history, with epilepsy (mostly drug-refractory epilepsy) as the main or the only clinical manifestation. The lesions of EAT are mostly located in or near the cortex, usually with no significant mass effect but accompanied by cystic changes and calcification, and most are WHO grade I-II tumors, which are curable after surgical resection. The common types of EAT include glioneuronal tumor and low-grade glioma [17]. EAT accounts for about 2-5\% of central nervous system tumors, and is the second most common cause of epilepsy surgery in adults (next to hippocampal sclerosis) and children (next to focal cortical dysplasia) [18]. In 2003, Luyken et al. proposed the concept of "long-term epilepsy-associated tumor", where "long-term" indicates having a long history of drug-resistant epilepsy of more than 2 years [4]. In this study, 2 cases of LEAT were included, 1 with dysembryoblastic neuroepithelial tumor found after a 17-year history of epilepsy, and 1 with gemistocytic astrocytoma after a 4-year history of epilepsy. As the development of imaging technology enables early discovery of some "secret" tumor lesions, the classification of LEAT alone has limited significance in clinical practice. However, brain imaging of EAT at early stage may not necessarily show the presence of tumor lesions, which can cause failure to diagnose and misdiagnose, therefore, it is of great significance to study the clinical characteristics of EAT.

EAT can be further subdivided into tumors of mixed neuronal and glial origin (glioneuronal tumor) and those of the glial origin based on histology. Glioneuronal tumors are composed of neurons and glia with different degrees of differentiation, have a poor proliferative ability and low malignancy, and are almost exclusively WHO grade I benign tumors, with a good prognosis and a low rate of recurrence and malignancy. Glioneuronal tumors commonly include ganglioglioma and embryonic dysplasia neuroepithelial tumors. The incidence of 
Table 5 Comparisons between EAT and non-EAT groups

\begin{tabular}{lllllllll}
\hline Group & $\begin{array}{l}\text { Male/ } \\
\text { Female } \\
(\boldsymbol{n})\end{array}$ & $\begin{array}{l}\text { Age } \\
\text { (years) }\end{array}$ & $\begin{array}{l}\text { Primary/ } \\
\text { Metastasis } \\
(\boldsymbol{n})\end{array}$ & $\begin{array}{l}\text { Glioma/other } \\
\text { types of } \\
\text { tumor }(\boldsymbol{n})\end{array}$ & $\begin{array}{l}\text { Involvement of } \\
\text { single/multiple } \\
\text { lobes }(\boldsymbol{n})\end{array}$ & $\begin{array}{l}\text { Involvement of one/ } \\
\text { both hemispheres } \\
(\boldsymbol{n})\end{array}$ & $\begin{array}{l}\text { Location in brain } \\
\text { parenchyma/ } \\
\text { meninges }(\boldsymbol{n})\end{array}$ & $\begin{array}{l}\text { Generalized/ } \\
\text { focal seizures } \\
(\boldsymbol{n})\end{array}$ \\
\hline EAT & $46 / 41$ & $\begin{array}{l}49.32 \pm \\
16.73\end{array}$ & $59 / 28$ & $46 / 41$ & $53 / 14$ & $56 / 11$ & $67 / 20$ & $50 / 37$ \\
& & & & & $28 / 21$ & $49 / 17$ & $42 / 24$ \\
Non- & $39 / 27$ & $56.65 \pm$ & $19 / 47$ & $11 / 55$ & $24 / 25$ & & & 0.69 \\
EAT & & 15.53 & & & & $<0.05$ & \\
$P$ & 0.44 & 0.06 & $<0.05$ & $<0.05$ & $<0.05$ & &
\end{tabular}

epilepsy in glioneuronal tumor as the first or the only manifestation is $\sim 80-100 \%$ [19]. Complex partial seizure, which is manifested as stunning, autonomous nervous system or mental symptoms, aura, automatism, etc., is the most common seizure type that occurs in $80 \%$ of ganglioglioma cases, followed by partial secondary generalized tonic-clonic seizures (50\%) [19]. Only a limited number of patients develop neurological deficits related to the tumor site, such as limb numbness, visual impairment, poor speech, and ataxia, as well as symptoms of elevated intracranial pressure such as headache, dizziness, nausea and vomiting when the tumor affects the cerebrospinal fluid circulation. The epilepsy-related low-grade gliomas originate from different glial cells, mainly belong to the WHO grade II tumors, and exhibit a diffuse infiltrative growth pattern. Although not having a high proliferation capacity, they can recur or become malignant, diagnosed mainly within the age range of 3045 years [20, 21]. Epilepsy can develop early as the most common major clinical manifestation, and also later in the course of the disease [10]. The seizures usually belong to the type of secondary generalized seizure [21]. Surgical treatment remains the most effective treatment for epilepsy-related low-grade gliomas. Drug resistance to AEDs is present in over $50 \%$ of patients before surgery, which may be attributed to insular involvement, delayed diagnosis, and location of tumor in functional areas [20]. In addition to epilepsy, the neurological symptoms of epilepsy-related low-grade gliomas also include headache, dizziness, elevated intracranial pressure, focal neurological deficits, cognitive decline, and mental abnormalities. The diagnosis of EAT mainly depends on

Table 6 Regression analysis of factors for EAT

\begin{tabular}{lll}
\hline Factor & Odd ratio & $\boldsymbol{P}$ \\
\hline Tumor type & 0.50 & 0.19 \\
Involvement of single/multiple lobes/meninges & 0.89 & 0.81 \\
Involvement of one/two hemispheres/meninges & 01.35 & 0.53 \\
Grade of glioma & & 0.01 \\
WHO I-II & 8.54 & \\
WHO III & 3.34 & \\
WHO IV & 1.44 & \\
\hline
\end{tabular}

clinical symptoms, imaging approaches and pathological examination. Diagnosis of EAT can be considered for refractory epilepsy based on abnormal computed tomography $(\mathrm{CT}) /$ magnetic resonance imaging (MRI) findings. More importantly, attentions should be paid to distinguish EAT from cortical dysplasia and intracranial inflammation. There are no clear clinical or imaging boundaries for distinguishing between EAT subtypes, so pathological examination is needed to confirm diagnosis. Immunohistochemical staining has the advantage of distinguishing between neurons and glial cells, and can facilitate histological classification of EAT. Given that the main clinical manifestation of EAT is epilepsy, electroencephalogram (EEG), especially long-term video electroencephalography (VEEG), is an important tool to evaluate EAT. EEG can provide guidance for locating and surgical resection of epileptic foci, evaluating disease severity, predicting the risk of epilepsy recurrence, and making adjustment of epilepsy medications. The interictal EEG signals of EAT patients do not have a specific pattern; they usually appear as spike waves and/or sharp waves, sometimes mixed with slow waves ipsilateral to the tumor. Some EEG activities can even be quite normal [9, 19]. In this study, the EAT patients had an average age of $49.32 \pm 16.73$ years, and the tumors were mostly gliomas, particularly WHO grade III gliomas, which are consistent with the literature. The EAT and non-EAT patients had no significant difference in the type of epilepsy or tumor location. This suggests the need of awareness for low-grade gliomas in epilepsy patients, especially drug-resistant epilepsy patients, who have unknown causes of epilepsy and a disease onset at the middle age. Moreover, follow-up observations, especially follow-up brain imaging, are important for distinguishing EAT from other causes of epilepsy, such as cortical dysplasia and inflammatory pathology. Brain biopsy examination should be performed if necessary.

\section{Conclusion}

In this study, we found that a majority of BTRE patients had tumor lesions located in the brain parenchyma, and most of them had generalized seizures. The type of seizure was related to the lobular distribution of the lesion, 
but not to the origin or the location (being in the brain parenchyma or the meninges) of tumor. In addition, "whether the tumor is glioma" and the glioma grade were determined to be independent factors for EAT, that is, the majority of EATs are gliomas, particularly lowgrade gliomas. However, as this study is limited by a small sample size, future in-depth studies with larger sample sizes are needed.

\section{Abbreviations}

BTRE: Brain tumor-related epilepsy; EAT: Epilepsy-associated tumor;

LEAT: Long-term epilepsy-associated tumor; WHO: World Health

Organization; EEG: Electroencephalogram; VEEG: Video

electroencephalography

\section{Acknowledgements}

Material support was provided by the Neurology Department of Mianyang Central Hospital. Expert technical assistance was provided by the Neurosurgery Department of Mianyang Central Hospital. Dr. Wei-jia Jiang from the West China Hospital, Sichuan University provided assistance in editing and reviewing the manuscript.

\section{Authors' contributions}

ZXW collected and analyzed data, and wrote the manuscript. ZLY collected and analyzed data. DJF supervised the study. LZP supervised the study. TYF supervised the study and revised the manuscript. The author(s) read and approved the final manuscript.

\section{Funding}

No

\section{Availability of data and materials}

The data generated and analyzed in the current study are available from the corresponding author upon reasonable written request.

\section{Ethics approval and consent to participate}

This retrospective study was approved by the Mianyang Central Hospital Research Ethics Board and they decided that the written informed consent was not necessary.

\section{Consent for publication}

Not applicable.

\section{Competing interests}

The authors declare that they have no competing interests.

\section{Author details}

'Department of Neurology, Mianyang Central Hospital, Mianyang 621000, China. ${ }^{2}$ Department of Neurosurgery, Mianyang Central Hospital, Mianyang 621000, China.

Received: 12 May 2020 Accepted: 13 November 2020

Published online: 30 December 2020

\section{References}

1. Maschio M. Brain tumor-related epilepsy. Curr Neuropharmacol. 2012;10(2): 124-33.

2. van Breemen MS, Wilms EB, Vecht CJ. Epilepsy in patients with brain tumours: epidemiology, mechanisms, and management. Lancet Neurol. 2007;6(5):421-30

3. Giulioni M, Martinoni M, Marucci G. Challenges in epilepsy-associated tumors. J Neurooncol. 2016:130(1):239-40.

4. Luyken C, Blümcke I, Fimmers R, Urbach H, Elger CE, Wiestler OD, et al. The spectrum of long-term epilepsy-associated tumors: long-term seizure and tumor outcome and neurosurgical aspects. Epilepsia. 2003;44(6):822-30.

5. Fisher RS, van Emde Boas W, Blume W, Elger C, Genton P, Lee P, et al. Epileptic seizures and epilepsy: definitions proposed by the International League Against Epilepsy (ILAE) and the International Bureau for Epilepsy (IBE). Epilepsia. 2005;46(4):470-2.
6. Scheffer IE, Berkovic S, Capovilla G, Connolly MB, French J, Guilhoto L, et al. ILAE classification of the epilepsies: Position paper of the ILAE Commission for Classification and Terminology. Epilepsia. 2017;58(4):512-21.

7. Louis DN, Perry A, Reifenberger G, von Deimling A, Figarella-Branger D, Cavenee WK, et al. The 2016 World Health Organization Classification of Tumors of the Central Nervous System: a summary. Acta Neuropathol. 2016; 131(6):803-20

8. Banerjee PN, Filippi D, Allen Hauser W. The descriptive epidemiology of epilepsy-a review. Epilepsy Res. 2009;85(1):31-45.

9. Giulioni M, Marucci G, Martinoni M, Marliani AF, Toni F, Bartiromo F, et al. Epilepsy associated tumors: Review article. World J Clin Cases. 2014;2(11): 623-41

10. Wang X, Yang Y, Liu YH, Mao Q. Progress in diagnosis, treatment and research of glioma-associated Epilepsy. Sichuan Med J. 2016;37(01):110-3.

11. Yu ZD, Lu JF, Wu JS. The research progress of the generation mechanism of glioma correlation of epilepsy. Chin J of Clin Neurosci. 2017;25(03):330336,360 .

12. Rudà R, Trevisan E, Soffietti R. Epilepsy and brain tumors. Curr Opin Oncol. 2010;22(6):611-20

13. Glantz MJ, Cole BF, Forsyth PA, Recht LD, Wen PY, Chamberlain MC, et al, Practice parameter: anticonvulsant prophylaxis in patients with newly diagnosed brain tumors. Report of the Quality Standards Subcommittee of the American Academy of Neurology. Neurology. 2000;54(10):1886-93.

14. Ibos G, Freedman DJ. Interaction between Spatial and Feature Attention in Posterior Parietal Cortex. Neuron. 2016;91(4):931-43.

15. Huang Z, Zhang J, Wu J, Qin P, Wu X, Wang Z, et al. Decoupled temporal variability and signal synchronization of spontaneous brain activity in loss of consciousness: An fMRI study in anesthesia. Neuroimage. 2016;124(Pt A): 693-703.

16. Sirven JI, Wingerchuk DM, Drazkowski JF, Lyons MK, Zimmerman RS. Seizure prophylaxis in patients with brain tumors: a meta-analysis. Mayo Clin Proc. 2004;79(12):1489-94

17. Zhang YX, Ding MP. Progress in clinical research on epilepsy-associated tumors. J Epilepsy. 2018;4(01):32-5.

18. Holthausen H, Blümcke I. Epilepsy-associated tumours: what epileptologists should know about neuropathology, terminology, and classification systems. Epileptic Disord. 2016:18(3):240-51.

19. Ertürk ÇÖ, Işler C, Uzan M, Özkara Ç. Epilepsy-related brain tumors. Seizure. 2017:44:93-7.

20. Pallud J, Audureau E, Blonski M, Sanai N, Bauchet L, Fontaine D, et al. Epileptic seizures in diffuse low-grade gliomas in adults. Brain. 2014;137(Pt 2):449-62.

21. You G, Sha ZY, Yan W, Zhang W, Wang YZ, Li SW, et al. Seizure characteristics and outcomes in 508 Chinese adult patients undergoing primary resection of low grade gliomas: a clinicopathological study. Neuro Oncol. 2012;14(2):230-41.

Ready to submit your research? Choose BMC and benefit from:

- fast, convenient online submission

- thorough peer review by experienced researchers in your field

- rapid publication on acceptance

- support for research data, including large and complex data types

- gold Open Access which fosters wider collaboration and increased citations

- maximum visibility for your research: over $100 \mathrm{M}$ website views per year

At BMC, research is always in progress.

Learn more biomedcentral.com/submissions 